$15^{\text {th }}$ International Conference on

AEROSPACE SCIENCES \& AVIATION TECHNOLOGY,

$\boldsymbol{A S A T}$ - 15 - May 28 - 30, 2013, Email: asat@mtc.edu.eg,

Military Technical College, Kobry Elkobbah, Cairo, Egypt,

Tel: +(202) 24025292 -24036138, Fax: +(202) 22621908

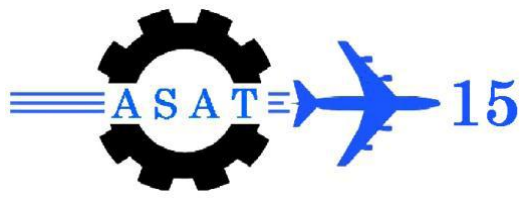

\title{
Transient Generalized Couette Flow with Heat Transfer of a Dusty Conducting Fluid with Ion Slip
}

\author{
$\{\text { H. A. Attia, K. M. Ewis }\}^{*}$
}

\begin{abstract}
In this study, the transient generalized Couette flow with heat transfer of a dusty viscous incompressible electrically conducting fluid is studied under the influence of a constant pressure gradient and considering the ion slip. An external uniform magnetic field is applied perpendicular to the plates which are kept at constant temperatures. A numerical solution for the governing momentum and energy equations are obtained using the method of finite differences. The influence of various magnetic field parameters (magnetic field intensity, Hall parameter, ion slip parameter) on the velocity and temperature fields of both the fluid and dust particles phases is demonstrated.
\end{abstract}

Keywords: Couette flow, heat transfer, dusty fluid, conducting fluid, ion slip effect

\section{Introduction}

The flow and heat transfer of conducting fluids through channels of different geometries in the presence of an external magnetic field have interesting applications in magnetohydrodynamic (MHD) generators, pumps, accelerators, and flow meters and has possible applcations in nuclear reactors, filtration, geothermal systems [1,2]. The existence of a dusty phase in the form of solid particles has important practical applications such as ash or soot in combustion MHD generators and plasma MHD accelerators [1,2].

The flow of dusty fluids was investigated by many authors [3-7]. The MHD flow of dusty fluids was also handled [8-12]. The Hall and ion slip effects were taken into considerations in applying generalized Ohm's law only in the case of strong magnetic fields $[13,14]$. The influence of the Hall current or the ion slip on the unsteady flow between parallel nonconducting plates, known as Hartmann flow, of a non-dusty fluid was examined by a number of researchers [15-19]. The influence of the Hall current on unsteady Couette flow of a dusty conducting fluid with heat transfer was studied by Attia [20].

In the present work, unsteady Couette flow with heat transfer of an electrically conducting, viscous, incompressible, dusty fluid is studied including both the Hall current and ion slip effects. The upper plate is moving with a constant velocity and the lower plate is fixed where the mass conservation is assumed. The fluid is flowing between two parallel infinite plates maintained at two constant but different temperatures. The fluid is acted upon by a constant pressure gradient and an external uniform magnetic field is applied perpendicular to the plates. The governing momentum and energy equations including the viscous and Joule dissipation terms are solved numerically using the method of finite differences to determine the velocity and temperature distributions for both the fluid and dust particles for various values of the parameters associated with the electromagnetic effect.

Department of Engineering Mathematics and Physics, Faculty of Engineering, Fayoum University, El-Fayoum-63514, Egypt. 


\section{Description of the Problem}

The dusty fluid is flowing between two parallel infinite horizontal plates located at the $y= \pm h$ planes. The upper plate is given with a constant velocity $U_{o}$ in the $x$-direction while the lower plate is fixed. The dust particles are assumed to be electrically non-conducting spherical in shape and uniformly distributed throughout the fluid. The two plates are assumed to be electrically non-conducting and kept at two constant temperatures $T_{1}$ for the lower plate and $T_{2}$ for the upper plate where $T_{2}>T_{1}$. A uniform and constant pressure gradient is applied in the $x$-direction and a uniform magnetic field $B_{o}$ is applied in the positive $y$-direction. Due to the inclusion of the Hall current term, $z$-components of the velocity for the fluid and dust particle phases arise. Due to the infinite dimensions in the $x$ - and $z$-directions, the physical quantities do not change in these directions and therefore we have $\partial / \partial x=\partial / \partial z=0$ and the problem becomes one-dimensional.

\section{The Velocity Distribution}

The momentum equation for fluid phase is given by

$\rho \frac{D v}{D t}=-\nabla P+\mu \nabla^{2} v+J x B_{o}-K N\left(v-v_{p}\right)$

where $\rho$ is the density of the fluid, $\mu$ is the viscosity of the fluid, $v$ is the velocity vector of the fluid, $\boldsymbol{v}=u(y, t) \boldsymbol{i}+w(y, t) \boldsymbol{k}, \boldsymbol{v}_{\mathrm{p}}$ is the velocity vector of dust particles, $\boldsymbol{v}_{\boldsymbol{p}}=u_{p}(y, t) \boldsymbol{i}+w_{p}(y, t) \boldsymbol{k}, \boldsymbol{J}$ is the current density, $N$ is the number of dust particles per unit volume, $K$ is the Stokes constant $=6 \pi \mu a$, and $a$ is the average radius of dust particles.

The last two terms in the right side of Eq. (1) are, respectively, the Lorentz force and the force associated with the relative motion between the fluid and dust particles. If the Hall and ion slip terms are retained, the current density $\boldsymbol{J}$ from the generalized Ohm's law is given by $[13,14]$

$J=\sigma\left(E+V x B_{o}-\beta\left(J x B_{o}\right)+\frac{\beta B i}{B_{o}}\left(J x B_{o}\right) x B_{o}\right)$

where $\sigma$ is the electric conductivity of the fluid, $\beta$ is the Hall factor and $B i$ is the ion slip parameter $[13,14]$. Solving Eq. (2) for $\boldsymbol{J}$ yields

$J x B_{o}=\frac{\sigma B_{o}^{2}}{(1+B i B e)^{2}+B e^{2}}\{((1+B i B e) u+B e w) i+((1+B i B e) w-B e u) k\}$

where $B e=\sigma \beta B_{o}$, is the Hall parameter [13,14]. In terms of Eq. (3), the two components of Eq. (1) read

$\rho \frac{\partial u}{\partial t}=-\frac{d P}{d x}+\mu \frac{\partial^{2} u}{\partial y^{2}}-\frac{\sigma B_{o}^{2}}{(1+B i B e)^{2}+B e^{2}}((1+B i B e) u+B e w)-K N\left(u-u_{p}\right)$

$\rho \frac{\partial w}{\partial t}=\mu \frac{\partial^{2} w}{\partial y^{2}}-\frac{\sigma B_{o}^{2}}{(1+B i B e)^{2}+B e^{2}}((1+B i B e) w-B e u)-K N\left(w-w_{p}\right)$

Applying Newton's second law applied in the $x$ and $z$-directions, gives the motion of the dust particles in the form

$m_{p} \frac{\partial u_{p}}{\partial t}=K N\left(u-u_{p}\right)$

$m_{p} \frac{\partial w_{p}}{\partial t}=K N\left(w-w_{p}\right)$

where $m_{p}$ is the average mass of dust particles. It is assumed that the pressure gradient is impulsively applied at $t=0$ and the fluid starts its motion from rest. Thus, 
$t \leq 0: u=u_{p}=w=w_{p}=0$

For $t>0$, the no-slip condition at the plates results in

$t>0, \quad y=-h: u=u_{p}=w=w_{p}=0$

$t>0, \quad y=h: u=U_{o}, u_{p}=w=w_{p}=0$

\section{The Temperature Distribution}

Heat transfer is from the upper hot plate to the lower cold plate by conduction through the fluid. There is no natural convection as the hot plate is above, however there is a forced convection due to the presence of suction and injection. Also there is a heat generation due to Joule and viscous dissipations. The dust particles gain heat from the fluid by conduction through their spherical surface [12,21]. The energy equations describing the temperature distributions for both the fluid and dust particles are respectively

$\rho c \frac{\partial T}{\partial t}=k \frac{\partial^{2} T}{\partial y^{2}}+\mu\left(\left(\frac{\partial u}{\partial y}\right)^{2}+\left(\frac{\partial w}{\partial y}\right)^{2}\right)+\frac{\sigma(1+B i B e) B_{o}^{2}}{(1+B i B e)^{2}+B e^{2}}\left(u^{2}+w^{2}\right)+\frac{\rho_{p} c_{s}}{\gamma_{T}}\left(T_{p}-T\right)$,

$\frac{\partial T_{p}}{\partial t}=-\frac{1}{\gamma_{T}}\left(T_{p}-T\right)$

where $T$ is the temperature of the fluid, $T_{p}$ is the temperature of the dust particles, $c$ is the specific heat capacity of the fluid at constant volume, $k$ is the thermal conductivity of the fluid, $\rho_{p}$ is the mass of dust particles per unit volume of the fluid, $\gamma_{T}$ is the temperature relaxation time, and $c_{s}$ is the specific heat capacity of the particles.

The last three terms on the right side of Eq. (9) represent the viscous dissipation, the Joule dissipation, and the heat conduction between the fluid and dust particles respectively. The temperature relaxation time depends on the geometry and since the dust particles are assumed to be spherical in shape, the last term in Eq. (9) is equal to $4 \pi a N k\left(T_{p}-T\right)$. Then

$\gamma_{T}=\frac{3 \operatorname{Pr} \gamma_{p} c_{s}}{2 c}$

where $\gamma_{p}$ is the velocity relaxation time $=2 \rho_{s} a^{2} / 9 \mu, \operatorname{Pr}$ is the Prandtl number $=\mu c / k$, and $\rho_{s}$ is the material density of dust particles $=3 \rho_{p} / 4 \pi a^{3} N$.

$T$ and $T_{p}$ must satisfy the initial and boundary conditions

$t \leq 0: T=T_{p}=T_{1}$,

$t>0, y=-h: T=T_{p}=T_{1}$,

$t>0, y=h: T=T_{p}=T_{2}$.

Introducing the following dimensionless variables and parameters

$(\hat{x}, \hat{y})=\frac{(x, y)}{h}, \hat{t}=\frac{t U_{o}}{h},(\hat{u}, \hat{w})=\frac{(u, w)}{U_{o}},\left(\hat{u}_{p}, \hat{w}_{p}\right)=\frac{\left(u_{p}, w_{p}\right)}{U_{o}}, \hat{P}=\frac{P}{\rho U_{o}^{2}}, \hat{T}=\frac{T-T_{1}}{T_{2}-T_{1}}, \hat{T}_{p}=\frac{T_{p}-T_{1}}{T_{2}-T_{1}}$

$\operatorname{Re}=U_{o} \rho h / \mu$, is the Reynolds number,

$H a=B_{o} h \sqrt{\sigma / \mu}$, the Hartmann number,

$E_{c}=U_{o}^{2} / c\left(T_{2}-T_{1}\right)$, the Eckert number,

$G=m_{p} \mu / \rho h^{2} K$, is the particle mass parameter,

$R=K N h^{2} / \mu$ is the particle concentration parameter.

$L_{o}=\rho h^{2} / \mu \gamma_{T}$ is the temperature relaxation time parameter. 
Equations (4)-(11) take the form (the hats are dropped for convenience)

$$
\begin{aligned}
& \frac{\partial u}{\partial t}=-\frac{1}{\operatorname{Re}} \frac{d P}{d x}+\frac{1}{\operatorname{Re}} \frac{\partial^{2} u}{\partial y^{2}}-\frac{H_{a}^{2}}{\operatorname{Re}\left((1+B i B e)^{2}+B e^{2}\right)}((1+B i B e) u+B e w)-\frac{R}{\operatorname{Re}}\left(u-u_{p}\right) \\
& \frac{\partial w}{\partial t}=\frac{1}{\operatorname{Re}} \frac{\partial^{2} w}{\partial y^{2}}-\frac{H_{a}^{2}}{\operatorname{Re}\left((1+B i B e)^{2}+B e^{2}\right)}((1+B i B e) w-B e u)-\frac{R}{\operatorname{Re}}\left(w-w_{p}\right) \\
& G \frac{\partial u_{p}}{\partial t}=u-u_{p} \\
& G \frac{\partial w_{p}}{\partial t}=w-w_{p} \\
& t \leq 0: u=u_{p}=w=w_{p}=0 \\
& t>0, y=-1: u=u_{p}=w=w_{p}=0 \\
& t>0, y=1: u=1, u_{p}=w=w_{p}=0 \\
& \frac{\partial T}{\partial t}=\frac{1}{\operatorname{Re} \operatorname{Pr}} \frac{\partial^{2} T}{\partial y^{2}}+\frac{E_{c}}{\operatorname{Re}}\left(\left(\frac{\partial u}{\partial y}\right)^{2}+\left(\frac{\partial w}{\partial y}\right)^{2}\right)+\frac{(1+B i B e) H_{a}^{2} E_{c}}{\operatorname{Re}\left((1+B i B e)^{2}+B e^{2}\right)}\left(u^{2}+w^{2}\right)+\frac{2 R}{3 \operatorname{Pr}}\left(T_{p}-T\right), \\
& \frac{\partial T_{p}}{\partial t}=-L_{o}\left(T_{p}-T\right), \\
& t \leq 0: T=T_{p}=0, \\
& t>0, y=-1: T=T_{p}=0, \\
& t>0, y=1: T=T_{p}=1 .
\end{aligned}
$$

Equations (12)-(19) represent a system of partial differential equations which is solved numerically using finite differences. The Crank-Nicolson implicit method [22] is used at two successive time levels where the finite difference equations relating the variables are obtained by writing the equations at the mid point of the computational cell and then replacing the different terms by their second order central difference approximation in the $y$-direction. The diffusion terms are replaced by the average of the central differences at two successive timelevels. Finally, the resulting block tri-diagonal system is obtained using generalized Thomasalgorithm [22]. Calculations are made for $d P / d x=5, R e=1, R=0.5, G=0.8, L_{o}=0.7, P r=1$, and $E_{c}=0.2$.

\section{Results and Discussion}

Figures 1-3 present the progression with time of the velocity components and temperature at the centre of the channel $(y=0)$, respectively, for the fluid and particle phases for various values of the Hall parameter $B e$ and the ion slip parameter $B i$ and for $H a=1$. It is apparent from Figs. $1 \mathrm{a}$ and $1 \mathrm{~b}$ that increasing the parameter $B e$ or $B i$ increases both $u$ and $u_{p}$ which can be attributed to the act that the effective conductivity $\left(\sigma /\left((1+B e B i)^{2}+B e^{2}\right)\right)$ decreases with increasing $B e$ or $B i$ which decreases the magnetic resistive force on $u$. In Figs. $2 \mathrm{a}$ and $2 \mathrm{~b}$, the velocity components $w$ and $w_{p}$ decrease with increasing $B e$ or $B i$ due to decreasing the source term of $w$ and increasing its damping term.

Figures $3 \mathrm{a}$ and $3 \mathrm{~b}$ show that, unless $B e$ and $B i$ are large, increasing $B e$ or $B i$ decreases $T$ and $T_{p}$ with times since an increase in $B e$ or $B i$ decreases the Joule dissipation which is proportional to $\left(1 /\left((1+B e B i)^{2}+B e^{2}\right)\right)$. On the other hand, for large values of $B e$ and $B i$, increasing $B i$ decreases $T$ and $T_{p}$ at small times and increases it at large times. This is because, for small times, $u$ and $w$ are small and an increment in $B e$ or $B i$ decreases the Joule 
dissipation which is also proportional to $\left(1 /\left((1+B e B i)^{2}+B e^{2}\right)\right)$. For large times, increasing $B e$ increases both $u$ and $w$ and, consequently increases the Joule and viscous dissipations. For large times, increasing $B i$, although it decreases $w$, it increases the velocity $u$ of the main flow and, in turn increases the viscous and Joule dissipations. This results in the crossing of the curves of $T$ with time for higher values of $B e$ and $B i$. The same observations are clear in Fig. $3 \mathrm{~b}$. Comparing Figs. $6 \mathrm{a}$ and $6 \mathrm{~b}$ emphasizes that the temperature of the fluid approaches the steady state faster than the temperature of the particles.

Figures 4-6 present the time development of the velocity components and temperature at the centre of the channel, respectively, for the fluid and particle phases for various values of the Hartmann number $\mathrm{Ha}$ and the ion slip parameter $B i$ and for $B e=3$. Figures $4 \mathrm{a}$ and $4 \mathrm{~b}$ indicate that the effect of $B i$ on $u$ and $u_{p}$ depends on $H a$. For small values of $H a$, a small increment of $B i$ decreases $u$ and $u_{p}$ as a result of increasing the resistive force on $u$ which is proportional to $B i$. Increasing $B i$ more increases the effective conductivity and, consequently reduces the resistive force on $u$ which increases both $u$ and $u_{p}$. However, for larger values of $H a, u$ becomes small, and therefore increasing $B i$ always decreases the effective conductivity which increases $u$ and $u_{p}$. It is also apparent that the effect of $B i$ on $u$ and $u_{p}$ becomes more pronounced for higher values of $\mathrm{Ha}$. Figures $5 \mathrm{a}$ and $5 \mathrm{~b}$ emphasizes that increasing the ion slip parameter $B i$ decreases $w$ and $w_{p}$ for all values of $H a$ and its effect is more clear for higher values of $H a$. It is interesting to detect an overshooting in the velocity components $u$ and $w$ for higher values of $H a$ and $B i$. Figures $6 \mathrm{a}$ and $6 \mathrm{~b}$ show that the effect of $B i$ on $T$ and $T_{p}$ depends on $H a$. For small values of $H a$ increasing $B i$ reduces $T$ and $T_{p}$ as a result of decreasing $u$ which decreases the dissipations. Increasing $B i$ more increases $T$ and $T_{p}$ as a result of increasing $u$ which increases the dissipations. However, for larger values of $H a, u$ and $w$ are small and increasing $B i$ decreases $T$ and $T_{p}$ as a result of decreasing the Joule dissipation. It is also detected that the effect of $B i$ on $T$ and $T_{p}$ becomes more pronounced for higher values of $\mathrm{Ha}$.

\section{Conclusion}

The transient generalized Couette flow with heat transfer of a dusty conducting fluid was studied considering the Hall and ion slip effects in the presence of uniform suction and injection. The influence of the magnetic field, the Hall parameter, and the ion slip parameter on the velocity and temperature distributions for both the fluid and particle phases was demonstrated. Increasing the ion slip parameter increases the velocity components for fluid and particle phases $u$ and $u_{p}$, but decreases the velocity components $w$ and $w_{p}$ and temperatures $T$ and $T_{p}$. For large values of the Hall parameter, the variation of $T$ and $T_{p}$ with the ion slip parameter was shown to depend on time. The influence of the Hall current on the velocity and temperature distributions decreases with increasing the ion slip. It is detected that the effect of the ion slip on the velocity components and temperatures of the fluid and dust particles becomes more apparent for higher values of $\mathrm{Ha}$ and the variation of the velocities $u$ and $u_{p}$ and temperatures $T$ and $T_{p}$ with the ion slip parameter depends upon the magnetic field.

\section{References}

1. Lohrabi, J., "Investigation of magnetohydrodynamic heat transfer in two-phase flow", Ph.D. Thesis, Tennessee Technological University, P.I., 1980.

2. Chamkha, A.J., "Unsteady laminar hydromagnetic fluid-particle flow and heat transfer in channels and circular pipes", International J. of Heat and Fluid Flow, Vol. 21, pp. 740-746, 2000.

3. Saffman, P.G., "On the stability of a laminar flow of a dusty gas," Journal of Fluid Mechanics, Vol. 13, p. 120, 1962. 
4. Gupta, R.K., and Gupta, S.C., "Flow of a dusty gas through a channel with arbitrary time varying pressure gradient," Journal of Applied Mathematics and Physics, Vol. 27, p. 119, 1976.

5. Prasad, V.R., and Ramacharyulu, N.C.P., "Unsteady flow of a dusty incompressible fluid between two parallel plates under an impulsive pressure gradient," Def. Sci . Journal, Vol. 30, p. 125, 1979.

6. Dixit, L.A., "Unsteady flow of a dusty viscous fluid through rectangular ducts," Indian Journal of Theoretical Physics, Vol. 28, No. 2, p. 129, 1980.

7. Ghosh, A.K. and Mitra, D.K., "Flow of a dusty fluid through horizontal pipes," Rev. Roum. Phys., Vol. 29, No. 631, 1984.

8. Singh, K.K., "Unsteady flow of a conducting dusty fluid through a rectangular channel with time dependent pressure gradient," Indian Journal of Pure and Applied Mathematics, Vol. 8, No. 9, p. 1124, 1976.

9. Mitra, P. and Bhattacharyya, P., "Unsteady hydromagnetic laminar flow of a conducting dusty fluid between two parallel plates started impulsively from rest," Acta Mechanica, Vol. 39, p. 171, 1981.

10. Borkakotia, K. and Bharali, A., "Hydromagnetic flow and heat transfer between two horizontal plates, the lower plate being a stretching sheet," Quarterly of Applied Mathematics, p. 461, 1983.

11. Megahed, A.A., Aboul-Hassan, A.L., and Sharaf El-Din, H., "Effect of Joule and viscous dissipation on temperature distributions through electrically conducting dusty fluid," Fifth Miami International Symposium on Multi-Phase Transport and Particulate Phenomena; Miami Beach, Florida, USA, Vol. 3, p. 111, 1988.

12. Aboul-Hassan, A.L., Sharaf El-Din, H., and Megahed, A.A., "Temperature due to the motion of one of them," First International Conference of Engineering Mathematics and Physics, Cairo, p. 723, 1991.

13. Crammer and Pai, Magnetofluid dynamics for Engineer and scientists, McGraw-Hill, 1973.

14. Sutton, G.W. and Sherman, A., Engineering Magnetohydrodynamics, McGraw-Hill, 1965.

15. Soundalgekar, V.M., Vighnesam, N.V., and Takhar, H.S., "Hall and Ion-Slip effects in MHD Couette flow with heat transfer," IEEE Transactions on Plasma Science, Vol. PS7, No. 3, Sept. 1979.

16. Soundalgekar, V.M. and Uplekar, A.G., "Hall effects in MHD Couette flow with heat transfer," IEEE Transactions on Plasma Science, Vol. PS-14, No. 5, Oct. 1986.

17. Attia, H.A., "Hall current effects on the velocity and temperature fields of an unsteady Hartmann flow," Can. J. Phys., Vol. 76, No.9, p. 739, 1998.

18. Aboul-Hassan, A.L. and Attia, H.A., "Hydromagnetic flow of a dusty fluid in a rectangular channel with Hall current and heat transfer," Can. J. Phys. Vol. 80, pp. 579589, 2002.

19. Attia, H.A., "Effect of the ion slip on the MHD flow of a dusty fluid with heat transfer under exponential decaying pressure gradient", Central European Journal of Physics (CEJP), Central European Science Journals, Vol. 3, No. 4, pp. 484-507, 2005.

20. Attia, H.A., "Hall effect on Couette flow with heat transfer of a dusty conducting fluid in the presence of uniform suction and injection", African Journal Of Mathematical Physics (AJMP), The Moroccan Grouping in High Energy Physics (GNPHE), Vol. 2, No. 1, pp. 97-110, 2005.

21. Schlichting, H., Boundary layer theory, McGraw-Hill, 1968.

22. Ames, W.F., Numerical solutions of partial differential equations, Second Ed., Academic Press, New York, 1977. 


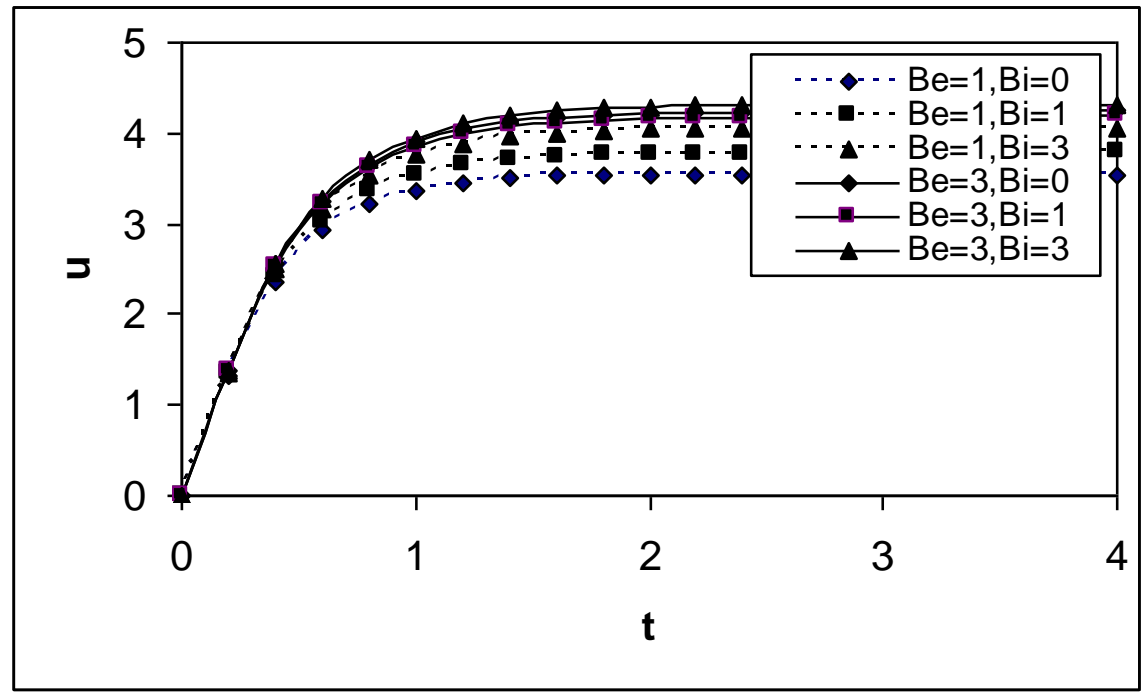

(a)

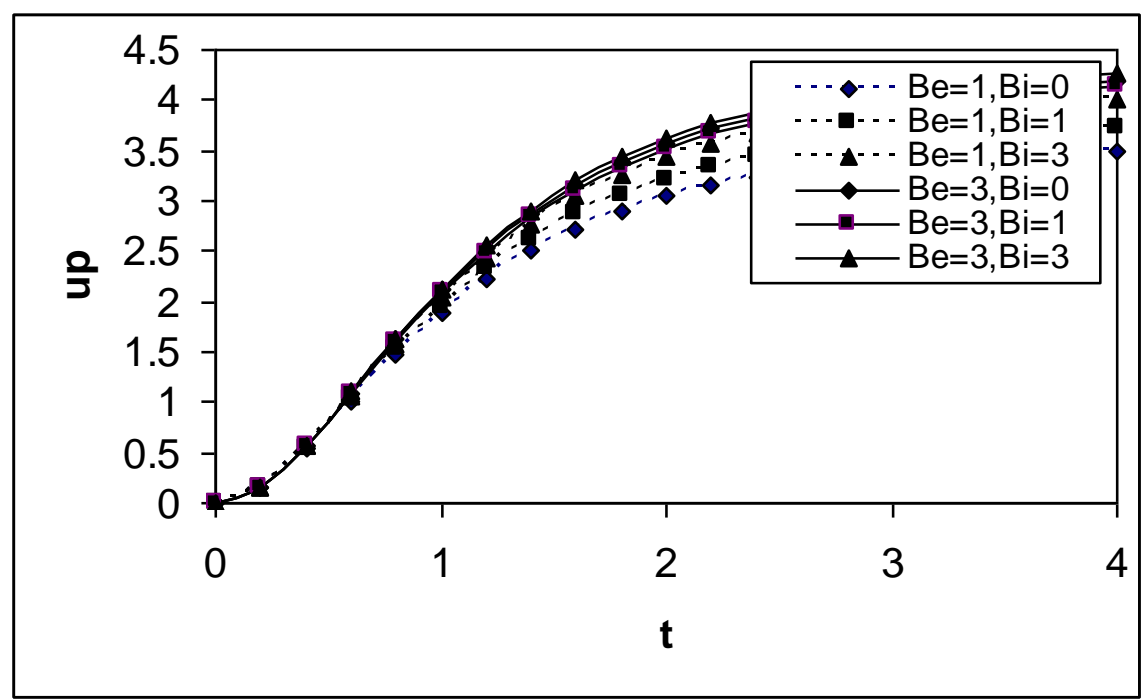

(b)

Fig. 1 Effect of the parameters $\mathrm{Be}$ and $\mathrm{Bi}$ on the time variation of: (a) $u$ at $y=0$ and (b) $u_{p}$ at $y=0$. (Ha=1) 


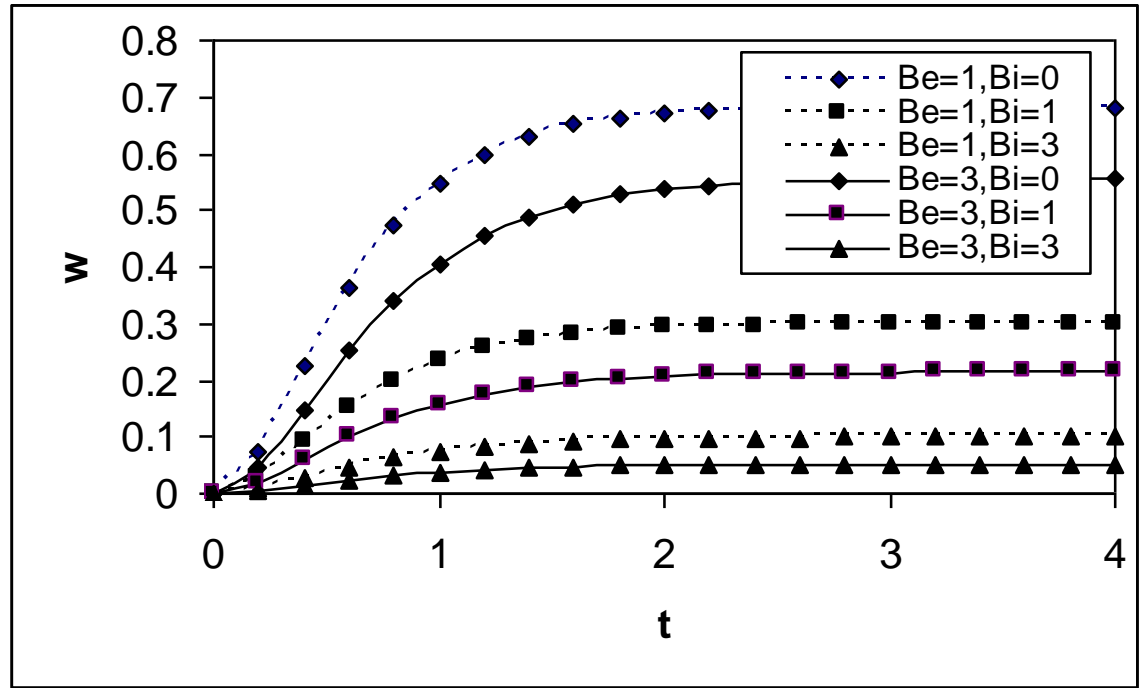

(a)

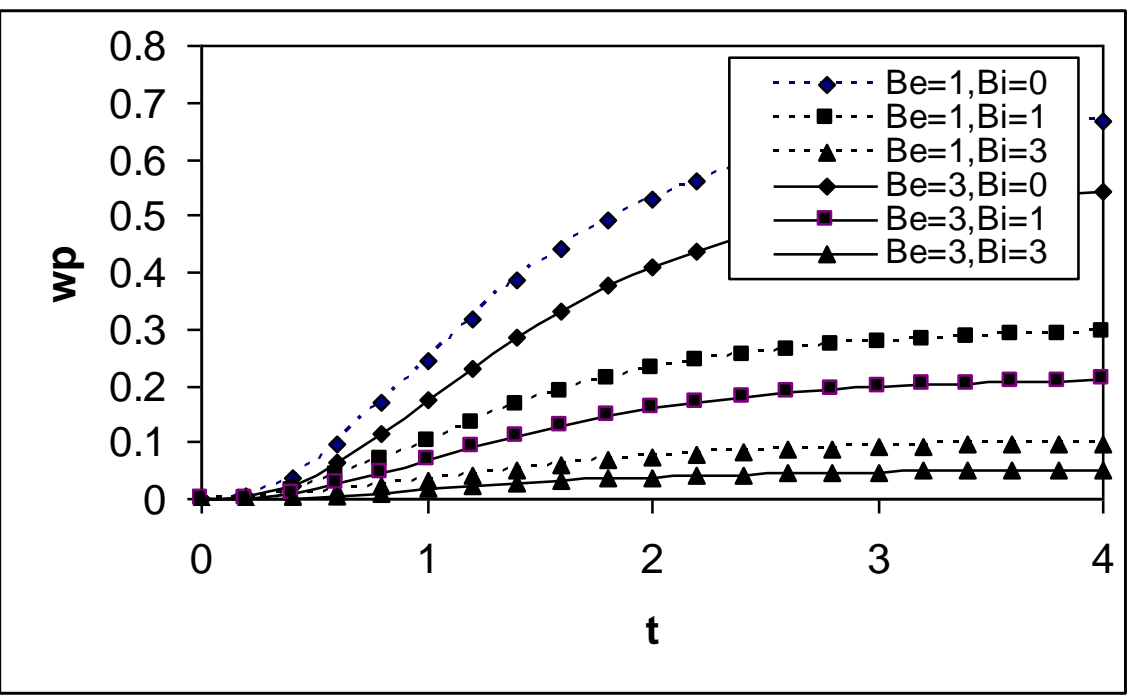

(b)

Fig. 2 Effect of the parameters $B e$ and $B i$ on the time variation of: (a) $w$ at $y=0$ and (b) $w_{p}$ at $y=0$. (Ha=1) 


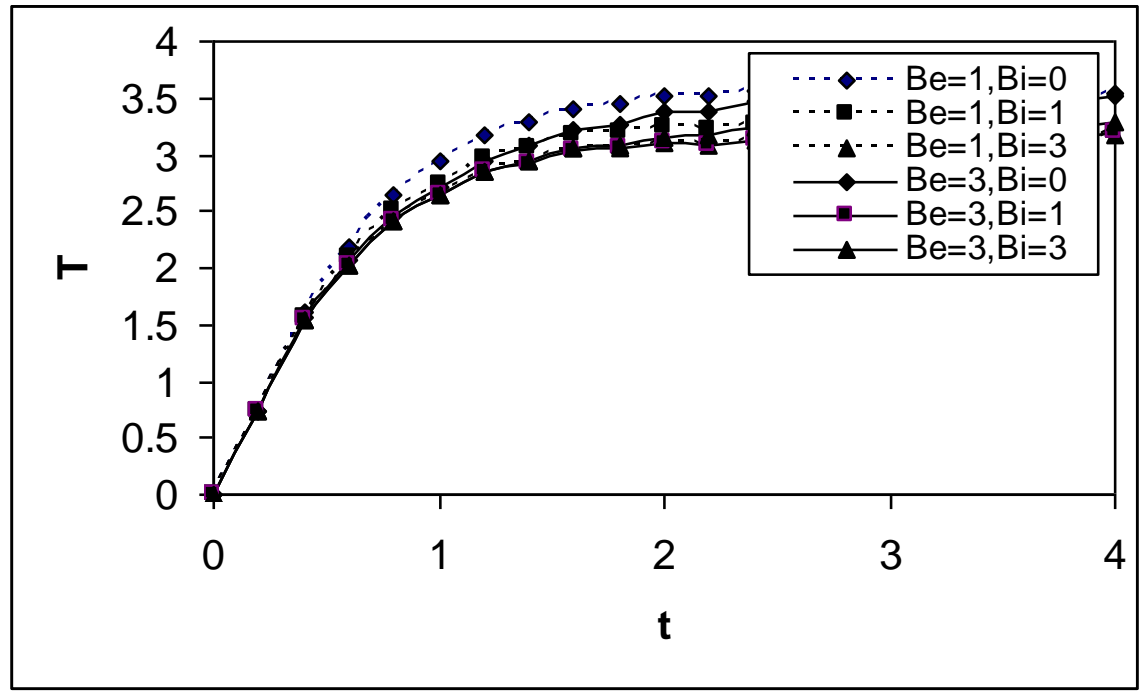

(a)

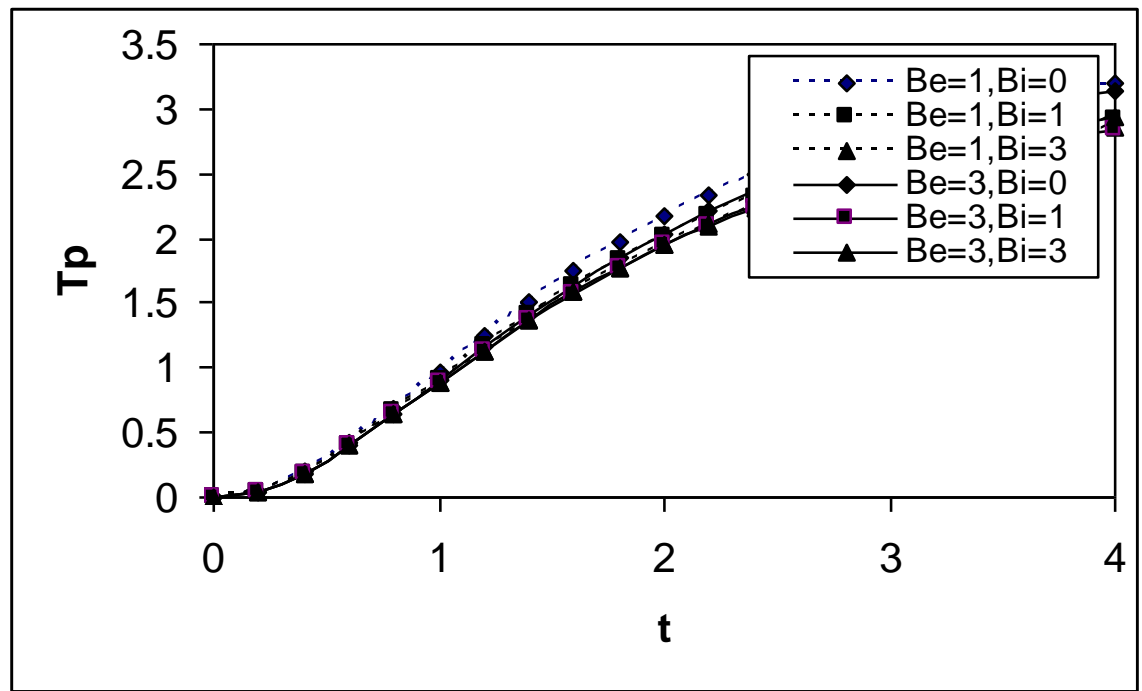

(b)

Fig. 3 Effect of the parameters $\mathrm{Be}$ and $\mathrm{Bi}$ on the time variation of: (a) $\mathrm{T}$ at $\mathbf{y}=0$ and (b) $\mathrm{T}_{\mathrm{p}}$ at $\mathbf{y}=0 .(\mathrm{Ha}=1)$ 


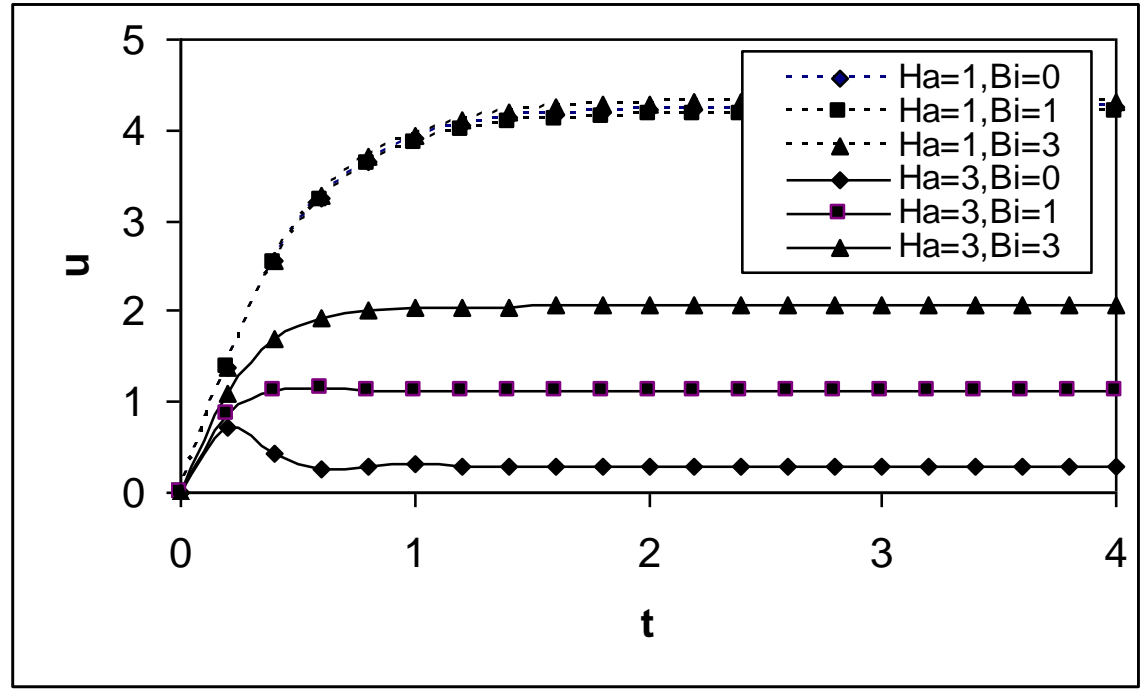

(a)

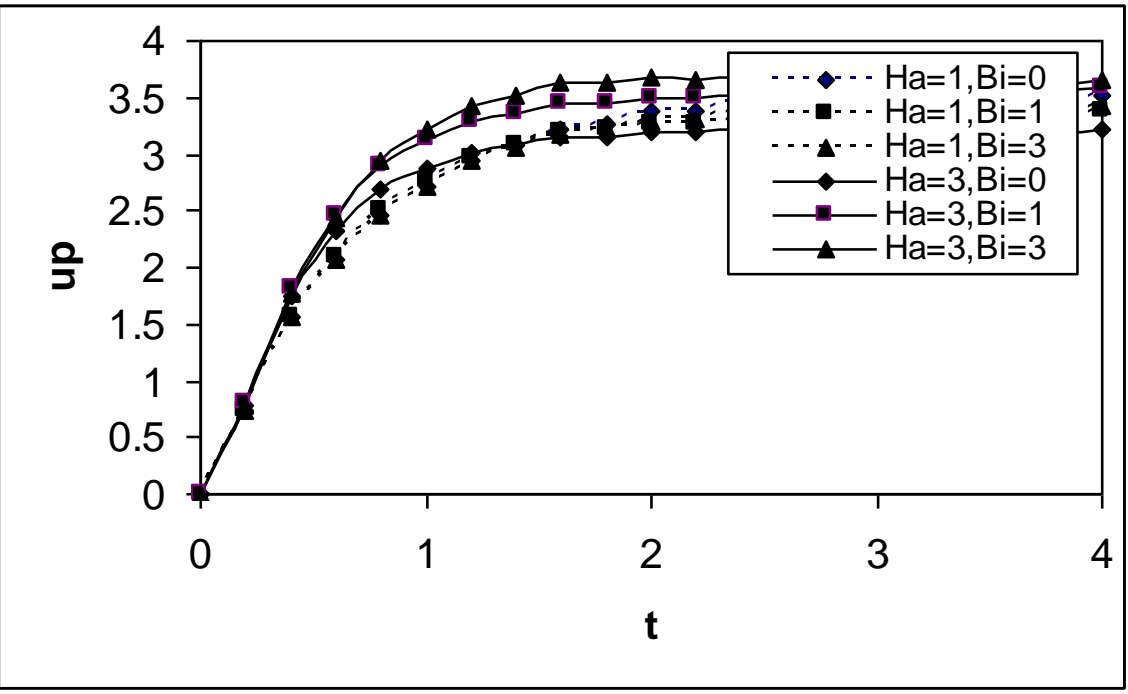

(b)

Fig. 4 Effect of the parameters $\mathrm{Ha}$ and $\mathrm{Bi}$ on the time variation of: (a) $u$ at $y=0$ and (b) $u_{p}$ at $y=0 .(B e=3)$ 


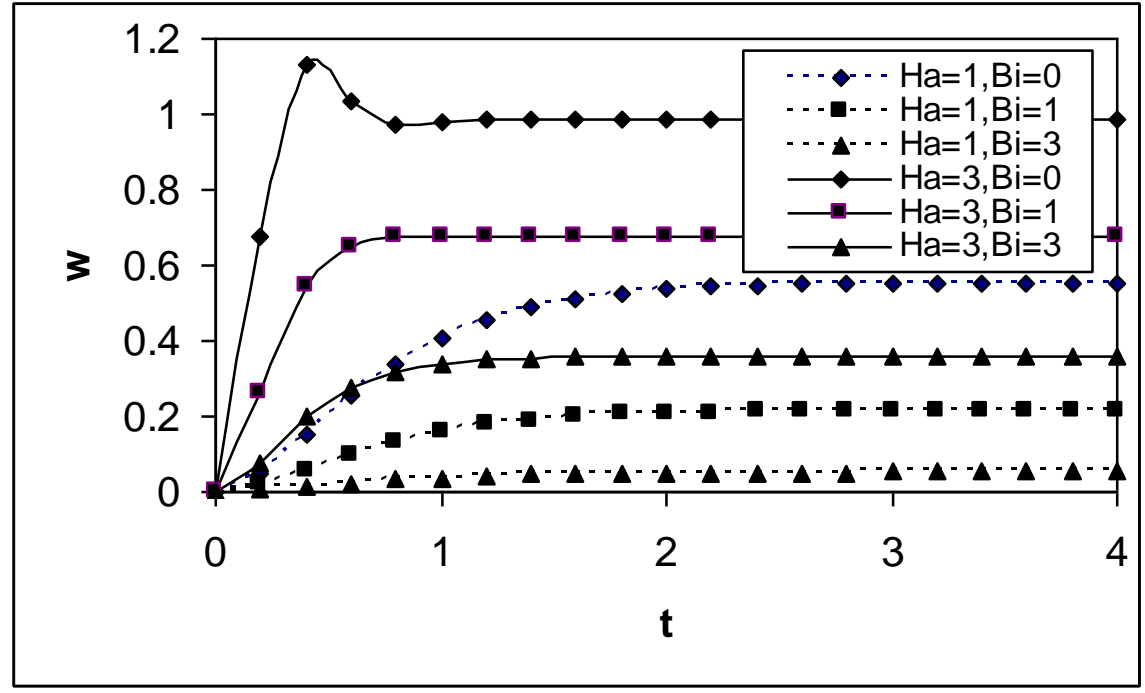

(a)

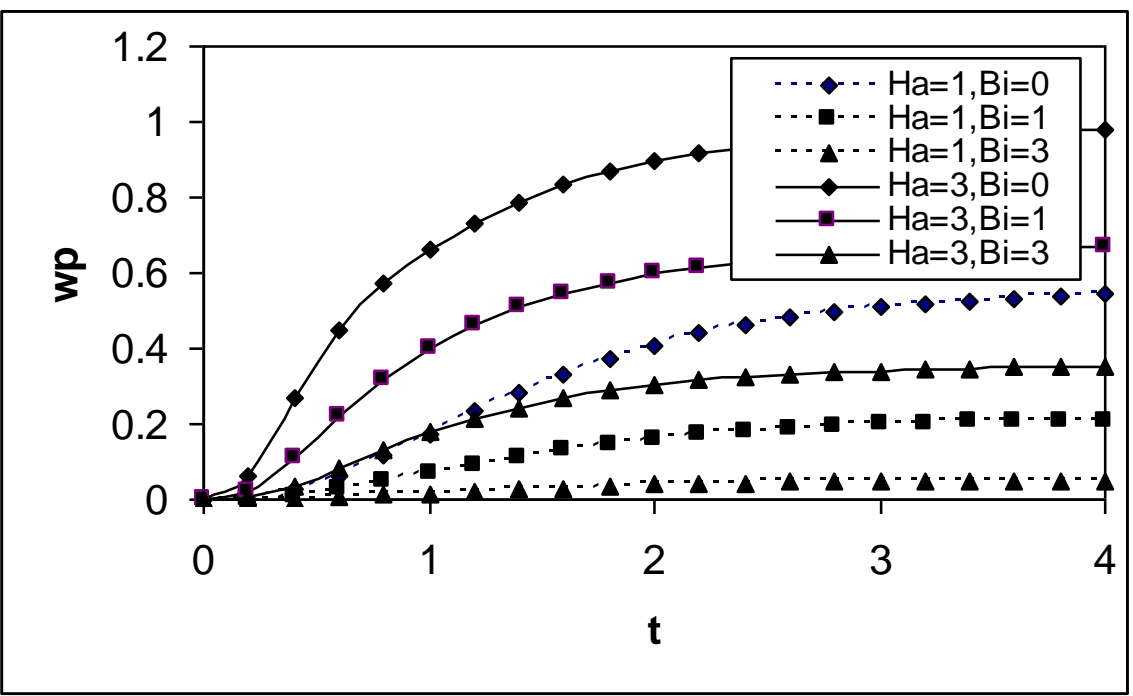

(b)

Fig. 5 Effect of the parameters $\mathrm{Ha}$ and $\mathrm{Bi}$ on the time variation of: (a) $w$ at $y=0$ and (b) $w_{p}$ at $y=0 .(B e=3)$ 


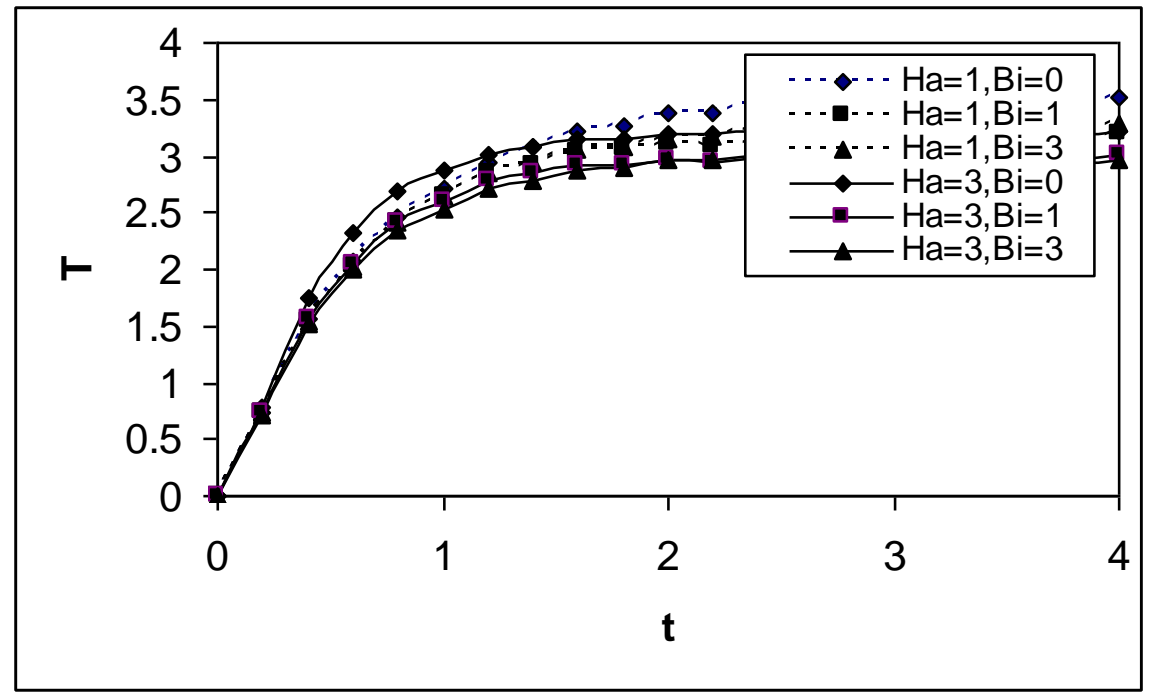

(a)

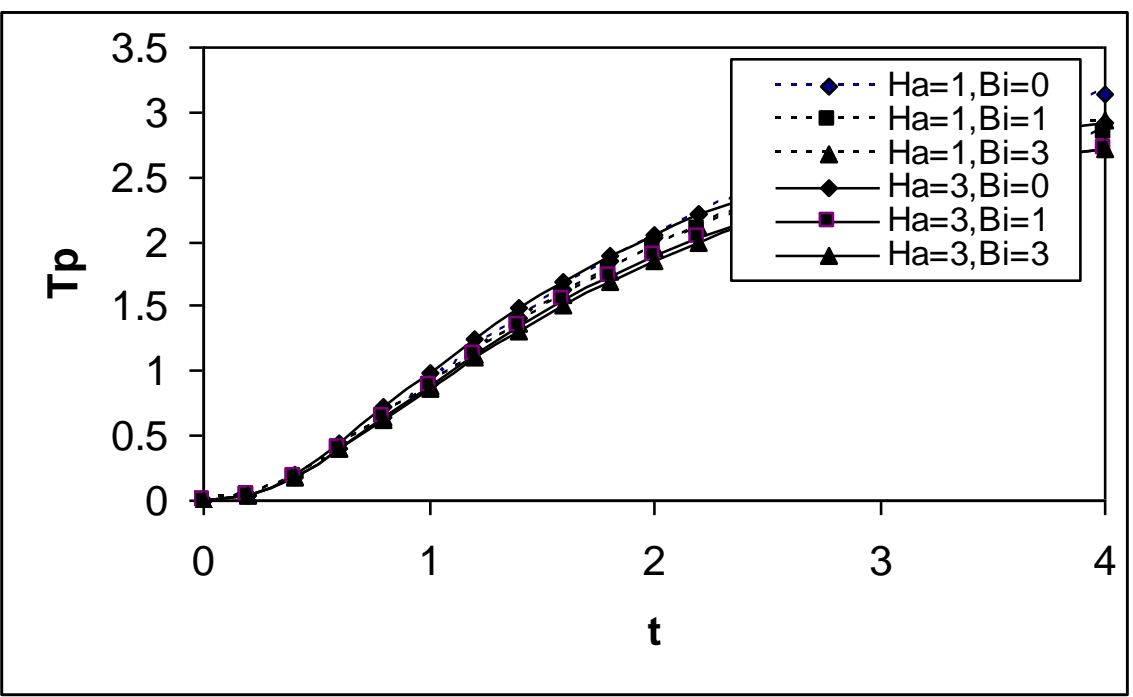

(b)

Fig. 6 Effect of the parameters $\mathrm{Ha}$ and $\mathrm{Bi}$ on the time variation of: (a) $T$ at $y=0$ and (b) $T_{p}$ at $y=0 .(B e=3)$ 